\title{
Salvia officinalis L. extract increase the antitumor effect of Doxorubicin on Ehrlich carcinoma tumor cells
}

\author{
Roxana Liana STAN ${ }^{1}$, Eleonora MARIAN ${ }^{2}$, Bogdan SEVASTRE ${ }^{3 *}$, Orsolya SÁRPATAKI ${ }^{3}$, Mariana \\ MUREȘAN $^{2}$, Alexandra Cristina SEVASTRE-BERGHIAN ${ }^{4}$, Tunde JURCA ${ }^{2}$, Adriana Corina HANGAN ${ }^{1}$ \\ ${ }^{1}$ University of Medicine and Pharmacy "Iuliu Hațieganu", Faculty of Pharmacy, Emil Isaac Street 13, \\ 400023, Cluj-Napoca, Romania \\ ${ }^{2}$ Faculty of Medicine and Pharmacy, University of Oradea, Nicolae Jiga Street 29, 410610, Oradea, Romania \\ ${ }^{3}$ University of Agricultural Science and Veterinary Medicine, Faculty of Veterinary Medicine, Mănăștur \\ Street 3-5, 400372, Cluj-Napoca, Romania \\ ${ }^{4}$ University of Medicine and Pharmacy "Iuliu Hațieganu", Faculty of Medicine, Emil Isaac Street 13, \\ 400023, Cluj-Napoca, Romania \\ *corresponding author: bogdan.sevastre@usamvcluj.ro
}

Bulletin UASVM Veterinary Medicine 76(1)/2019

Print ISSN 1843-5270; Electronic ISSN 1843-5378

doi:10.15835/buasvmcn-vm: 2019.0009

\begin{abstract}
The current study demonstrated the superior in vivo antitumor activity of the association between Salvia officinalis L. and Doxorubicin as compared with Doxorubicin alone and its lack of toxicity. The study was carried out on 32 Mus musculus female mice, for 14 days long. The tumor model was Ehrlich Ascites Carcinoma (EAC). Animals were divided in four equal groups of 8 mice: normal control, tumor control, EAC + doxorubicin and EAC + doxorubicin + Salvia officimalis L. All the values that reflect the tumor development (difference in body weight, EAC volume and cellular concentration) were improved in significant manner. Hematological and biochemistry parameters determination were performed for all four groups of mice and no cytotoxicity was found. The efficacy of Salvia officinalis L. as an adjuvant therapy in cancer has been demonstrated.
\end{abstract}

Key words: Salvia officinalis, mice, Ehrlich ascitic carcinoma, Doxorubicin, antitumor

\section{Introduction}

Despite some progress, cancer remains a major research challenge. Nowadays, research for new anticancer drugs is a very active domain (Sevastre et al., 2017). Plants have played an important role as a source of effective anticancer agents and it is significant that over $60 \%$ of currently used anticancer agents are derived in one way or another from natural sources including plants, marine organisms and microorganisms (Kamatou et al., 2008). Nature contains more than 1000 species of plants with significant anticancer properties. The investigation of natural anticancer substances from plants and herbs was initiated in 1960s, when vincristine, vinblastine, camptothecin and taxol were discovered (Tamrat and Yesudass, 2018).

Salvia officinalis L. (S. officinalis) is an odorous plant that is endemic to southern Europe and Asia and is widely used for both dietary and medicinal composition. Salvia is the largest genus of the Lamiaceae family, with around 900 species described worldwide. It has different biological activities that are manifested by its different components, and this "panacea" reputation is often just linked to its essential oils, even if its chemical composition is different due to genetic aspects and environmental factors (Privietera et al., 2019). Previous studies have demonstrated that the active principles in Salvia officinalis are the phenolic 
compounds: caffeic acid, carnosic acid, carnosol, kaempferol, oleanolic acid, rosmarinic acid and ursolic acid and the essesntial oils: $\alpha$-pinene, 1,8-cineole, linalool, limonene, borneol, myrcene, $\beta$-caryophyllene, spathulenol, $\beta$-caryophyllene oxide, viridiflorol, $\delta$-3-carene and $\alpha$-bisabolol (Kamatou et al., 2008). Due to its composition in these active principles, Salvia officinalis has been shown to have antibacterial, fungistatic, virustatic, astringent, antiinflamatory, antiplasmodial, antiooxidant and anticancer activities (Keshavarz et al., 2011; Fiore et al., 2006).

The current study had two objectives. First, was to demonstrate the in vivo antitumor effect of Salvia officinalis L. harvested from Romania and the fact that the association between Salvia officinalis L. and Doxorubicin increase the antitumor effect of Doxorubicin. The second objective was to follow the ability of Salvia officinalis L. to achieve protection against the side effects caused by the tumor grouth and to demonstrate its lack of toxicity. The study is a follow up of an in vitro study performed also on Salvia officinalis L. when we demonstrated its antioxidant and antitumor properties (Marian et al., 2018).

\section{Materials and methods Plant material and preparation of the extract}

The leaves of Salvia officinalis L. (Salviae folium) were harvested from Bihor region, Romania, from May to June from crops untreated with herbicides substances. After harvesting plant products were dried at the room temperature (20 $-25^{\circ} \mathrm{C}$ ), away from light.

For the extraction of bioactive compounds from the plant product, a $70 \%$ hydroalcoholic mixture $(1: 10 \mathrm{~g} / \mathrm{v})$ was used as an extraction solvent. The extraction mixture was subjected to a shaking operation using a magnetic homogenizer for 20 minutes followed by sonication for 5 minutes (Romanian Pharmacopoeia $10^{\text {th }}$ edition). At the end of the extraction, the hydroalcoholic extracts were subjected to centrifugation and the supernatants evaporated to dryness in a rotary evaporator. The dry extract was dispersed in 10 $\mathrm{mL}$ distilled water and then frozen at $-25^{\circ} \mathrm{C}$. Finally, they were brought to the lyophilizer and the lyophilized extracts were weighed and transferred to a dry sealed container.

\section{Animal care and experimental procedures}

The experiments on animals were performed according to the Directive 2010/63/EU and National low no. 43/11.04.2014. The study was performed into the Establishment for the breeding and use for laboratory animals of the University of Medicine and Pharmacy "IuliuHatieganu" Cluj-Napoca; the experiment was approved by The Bioethical Board of UMF "IuliuHațieganu" Cluj-Napoca, and authorized by state veterinary authority. The animals were caged in polycarbonate cages, at controlled temperature (of $21-22^{\circ} \mathrm{C}$ ), humidity (40-60\%) and $12 / 12 \mathrm{~h}$ light/ dark cycle. Standard lab chow, provided by the National Institute for Research and Development Cantacuzino Bucharest, and water were freely available. Mus musculus female mice with a body weight about $33.55 \pm 3.43$ g were used in the study. The mice were organized in four equal groups of eight mice and treated as follows:

Group I - normal control group, receiving placebo therapy intraperitoneally $(0.5 \mathrm{ml}$ saline physiological solution / mice, i.p);

Group II - tumor control group (EAC). Mice implanted with Ehrlich ascites carcinoma cells, $10^{6}$ ascitic cells each, on day 0 , and placebo therapy intraperitoneally $(0.5 \mathrm{ml}$ saline physiological solution / mice, i.p);

Group III - EAC and doxorubicin administration. Accordingly to manufacturer specification, the $\mathrm{LD}_{50}$ for doxorubicin chloride, in mice, in intraperitoneally administration, is $21.9 \mathrm{mg} /$ $\mathrm{kg}$. The normal therapeutically dose being approximately 10 times lower, during the present experiment, mice received $2.5 \mathrm{mg}$ doxorubicin chloride/b.w. (Adriblastina $10 \mathrm{mg}$, Pfizer) dissolved in sterile saline solution, up to $0.5 \mathrm{ml}$ per animal. The mentioned doses were administered in the $1^{\text {st }}$ and $6^{\text {th }}$ day of the experiment.

Group IV - EAC and doxorubicin + Salvia officinalis L. administration. Doxorubicin was administered under the same conditions. $50 \mathrm{mg}$ d.s. Salvia officinalis L. / b.w. was administrated intraperitoneally in the $1^{\text {st }}, 3^{\text {rd }}$ and $6^{\text {th }}$ day, in 0.5 $\mathrm{ml}$ sterile saline solution per animal. The dose has been established following preliminary toxicity studies.

14 days after EAC implantation, blood samples were collected by retro-orbital puncture under slight anesthesia condition, then, euthanasia was 
induced by prolonged narcosis. Blood hematology (complete blood count) was investigated with Abacus Junior Vet, Diatron, 3 Diff Messtechnik, Hungary. Blood chemistry (urea, creatinine and transaminase) was measured using screen point semiautomatic analyzer, STAT - FAX 1904 Plus, Global Medical Instrumentation, Inc. 6511 Bunker Lake Blvd. Ramsey Minesota, 55303 USA by using special determination kits (Diagnosticum Zrt. Hungary).

The volume of ascitic fluid from the peritoneal cavity was collected and measured with a syringe, immediately after euthanasia, and transferred in phosphate buffer solution $\left(\mathrm{pH} 7.4\right.$, at $\left.4^{\circ} \mathrm{C}\right)$. Then, the samples were subjected to repeated centrifugations (at $4^{\circ} \mathrm{C}$ ), first at $4.500 \mathrm{rpm}$ for $5 \mathrm{~min}$, then at $12.000 \mathrm{rpm}$ for $3 \mathrm{~min}$, in order to obtain a dense cell suspension, which was also stored at deep freezer until further use. The tumor cell concentration was counted in a Burker chamber (dilution 1:10) and the cell viability was assessed by Tripan blue staining ( $0.4 \%$ in PBS). Body weight was measured at the beginning, in the days 3, 6, 9, 12 and at the end of the study. At the end of the study the survival rate was $100 \%$ (Stan et al., 2013; Sevastre et al., 2014; Sarpataki et al., 2016).

\section{Statistical analysis}

All data are reported as the mean \pm SEM. The Gaussian distribution was checked by the Shapiro-Wilk normality test. One-way analysis of variance ANOVA, followed by post hoc Dunnett's range test procedure was performed for pair-wise comparisons between the volume of ascitic fluid and variation of viable cells concentration, while the two-way ANOVA followed by the Bonferroni post-test was the choice for the variation of body weight. Statistical significance was at $\mathrm{p}<0.05(95 \%$ confidence interval). Statistical values and figures were obtained using GraphPad Prism version 5.0 for Windows, GraphPad Software, San Diego California USA.

\section{Results and discussion}

The first objective of this study was to demonstrate the in vivo antitumor effect of Salvia officinalis L. harvested from Romania and the fact that the association between Salvia officinalis L. and doxorubicin increase the antitumor effect of doxorubicin.
The body weight, as a measure of Ehrlich ascites accumulation in the peritoneal cavity is shown in Fig.1. As expected, the ascites development was followed by the increase in the body weight. The doxorubicin and doxorubicin + Salvia officinalis L. therapies prevents body weight gain. The differences were visible starting from the third day of the study. Both therapies proved to provide significant protection, but the effect was better in the group treated with doxorubicin + Salvia officinalis L.

The amount of ascitic fluid has varied proportionally with the body weight gain. It has been found a significant decrease in the ascitic volume (Fig.2A) and in the tumor cell concentration (Fig.2B) for both EAC + doxorubicin and EAC + doxorubicin + Salvia officinalis L. groups as compared with the tumor control group. For the $\mathrm{EAC}+$ doxorubicin + Salvia officinalis L. group the decrease was more significant compared to the EAC + doxorubicin group. This demonstrated the effectiveness of associating the plant extract with doxorubicin.

Tumor development was prevented by doxorubicin therapy in a significant manner. The association between doxorubicin and Salvia officinalis L. provide a better antiproliferative effect as compared to doxorubicin alone. In fact, all the values that reflect the tumor development (difference in body weight, EAC volume and cellular concentration) were improved in significant manner (Figures 1, 2 A, B).

The mechanism of action for doxorubicin is still unclear, but is widely accepted an antiproliferative mechanism based on intercalation on DNA molecule, and consequently by blocking the DNA biosynthesis (Thorn et al., 2011).

Antitumor activity of Salvia officinalis L. is due to its anti-angiogenic, anti-migratory and anti-proliferative effects (Keshavarz et al., 2011). Most primary solid tumors are dependent on angiogenesis for survival, growth, invasion and metastasis. Therefore, targeting the angiogenesis process has become one of the important strategies in the treatment and prevention of cancer progression (Pandya et al., 2006). In the angiogenesis process, vascular endothelial cells migrate out from the parental vessels, invade through matrix, proliferate and form capillary tubes (Folkman, 2006). Anti-angiogenic agents were known to inhibit proteases, suppress receptor 


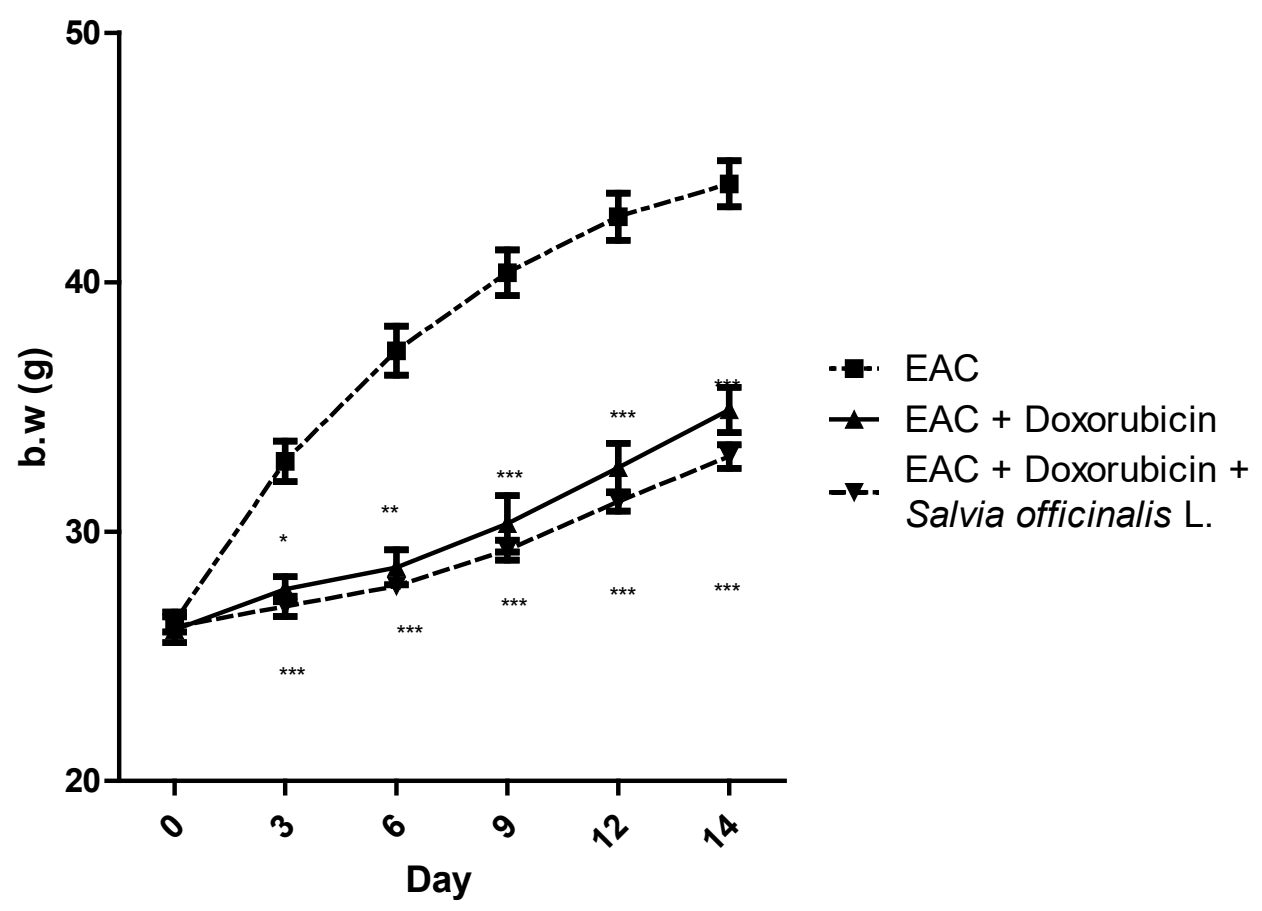

Figure 1. Effects of doxorubicin and of the association between doxorubicin and Salvia officinalis L. on the body weight gain $(\mathrm{g}) \mathrm{C}^{*}=\mathrm{p}<0.05:{ }^{* *}=\mathrm{p}<0.01 ;{ }^{* *}=\mathrm{p}<0.001$ as compared to EAC group) (mean \pm SEM) (8 animals/group)

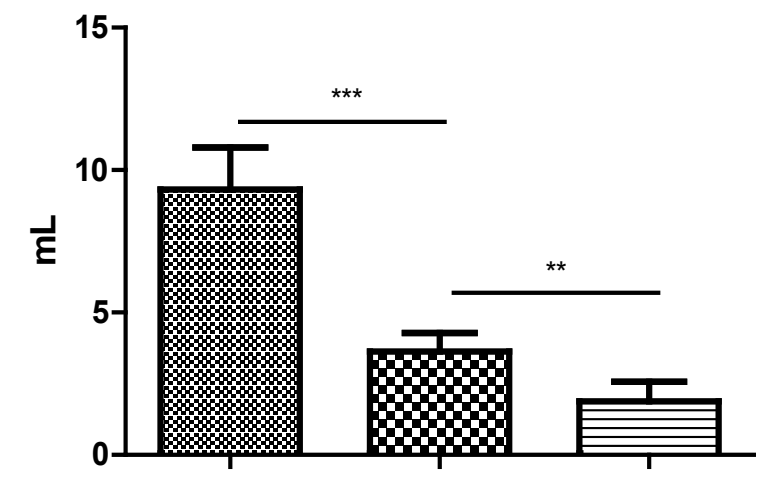

A
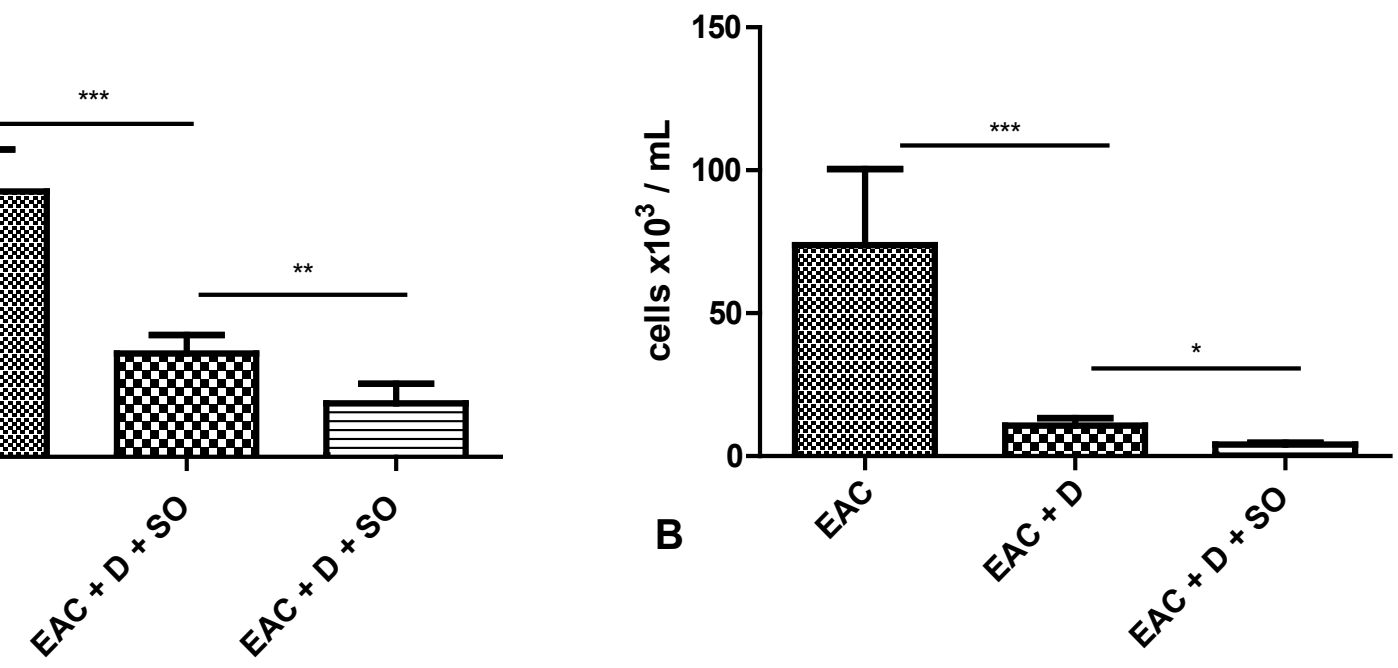

Figure 2. Effects of doxorubicin and of the association between doxorubicin and Salvia officinalis L. on ascitic volume $(\mathrm{ml})(\mathbf{A})$ and tumor cell concentration (cells x103/ $\mathrm{mL})(\mathbf{B})$.

$\left({ }^{*}=\mathrm{p}<0.05 ;{ }^{* *}=\mathrm{p}<0.01 ;{ }^{* * *}=\mathrm{p}<0.001\right.$ as compared to EAC group) (mean \pm SEM) (8 animals/group)

phosphorylation or disrupt endothelia tube formation (Murray, 2001). Ursolic acid isolated from Salvia officinalis L., significantly inhibited some proteases as included in tumor invasion and metastasis to combat with the pathologies including cancer. Ursolic acid could be one of the potent candidates which affected endothelial cell migration (Jedinak et al., 2006). Anti-angiogenic activity of Salvia officinalis L. extract is due to anti-migratory more than anti-proliferative effect, anti-migratory activity are probably attributed to anti-protease and/or interference of cytoskeleton 
organization, which are known to play important roles in cell locomotion and capillary tube formation (Plank and Sleeman, 2003).

As a conclusion of the first part of the study, the association between doxorubicin and Salvia officinalis L. enhance the antitumor effect of doxorubicin administrated alone.

The second objective was to follow the ability of Salvia officinalis L. to achieve protection against the side effects caused by the tumor grouth and to demonstrate its lack of toxicity. For this purpose, the hematological and biochemical parameters were determined.

The development of EAC was associated with a mild anemia, the red blood cells and the hematocrit were significant below control (Tab.1), but the red cells indices were in normal limits.

In EAC inoculates animals, the administration ofd oxorubicin and of doxorubicin + Salvia officinalis L. it seems to maintain the RBC level significant higher than those found in EAC group. The RBC level for EAC + Doxorubicin + Salvia officinalis L. group is superior to EAC + Doxorubicin group. The explication is still unknown, the protective effect seems to be rather indirect than a direct action on RBC: the degree of anemia is related to tumor development, so by preventing the EAC growth, the administration of Doxorubicin and of Doxorubicin + Salvia officinalis L. prevents also the progression of anemia (Tab.1).

EAC development was followed by significant leukocytosis. Granulocytes and middle cells were also increased in a significant manner. In EAC inoculated groups, doxorubicin therapy lowers WBC count as compared to untreated EAC group, but the value remains much higher than the control.
The association doxorubicin + Salvia officinalis L. in EAC inoculated group was responsible for a significant reduction of WBC as compared to EAC inoculated group, superior to the reduction of WBC determined by the administration of Doxorubicin alone. All leucocyte categories were involved in the same way (Tab.2). The association between Doxorubicin and Salvia officinalis L. may have an immunosuppressive effect, but the prevention of leukocytosis also might be due to the reduction of tumor growth.

The total platelet count (PLT), platelet hematocrit (PCT), mean platelet volume (MPV) and distribution width (PDWs) do not undergo significant statistical changes compared to control and EAC group and there was no evidence of toxicity. Neither the biochemistry parameters (creatinine, urea, transaminase) do not show significant statistical changes compared to control and to EAC group, during the two weeks of the current study.

In this way we demonstrated that the association of Salvia officinalis L. to Doxorubicin provides some protection to the hematological parameters and it's not toxic.

\section{Conclusion}

Doxorubicin and Salvia officinalis L. combined therapy seems to increase the antiproliferative effect against experimental inoculated cancer cells (EAC), without additional cytotoxicity. These facts highlight Salvia's usefulness as adjuvant treatment of cancer. The plant was harvested from spontaneous flora of Romania, so in the future we could prepare a native phytoproduct with utility

Table 1. The effect of association between Salvia officinalis L. extract and doxorubicin on red blood cells (RBC), hemoglobin (HGB) and hematocrit (HCT) (mean \pm S.E.M.)

\begin{tabular}{lccc}
\hline \multicolumn{1}{c}{ Group } & RBC $\mathbf{1 0}^{\mathbf{1 2}} / \mathbf{l}$ & HGB g/dl & HCT \% \\
\hline Control & $8.19 \pm 0.40$ & $12.56 \pm 1.32$ & $37.21 \pm 1.05$ \\
\hline EAC & $6.41 \pm 0.37^{*}$ & $10.46 \pm 1.05$ & $30.73 \pm 1.15^{*}$ \\
\hline EAC + Doxorubicin & $7.13 \pm 0.68$ & $10.78 \pm 0.80$ & $33.46 \pm 2.54$ \\
\hline EAC + Doxorubicin + S. officinalis L. & $7.49 \pm 0.50$ & $11.95 \pm 0.72$ & $35.81 \pm 2.63$ \\
\hline
\end{tabular}

Normal values: RBC 7-12.5 1012/l HGB 10.2-18 g/dl HCT 36-49 \% (Uray, 1992)

$C^{*}=\mathrm{p}<0.05$ as compared to control group) 
Table 2. The effect of association between Salvia officinalis L. extract and doxorubicin on white blood cells (WBC), lymphocytes (LYM), middle cells (MID) and granulocytes (GRA)

\begin{tabular}{|c|c|c|c|c|}
\hline Group & WBC $10^{9} / \mathbf{l}$ & LYM $10^{9} / 1$ & MID $10^{9} / 1$ & GRA $10^{9} / \mathbf{l}$ \\
\hline Control & $8.62 \pm 1.83$ & $4.29 \pm 0.65$ & $0.19 \pm 0.07$ & $1.47 \pm 0.26$ \\
\hline EAC & $27.22 \pm 2.32^{*}$ & $5.17 \pm 0.97$ & $0.76 \pm 0.15^{*}$ & $15.05 \pm 2.79^{*}$ \\
\hline EAC + Doxorubicin & $17.01 \pm 2.16^{\mathrm{a}}$ & $4.83 \pm 0.88$ & $0.37 \pm 0.11$ & $7.61 \pm 2.20^{\mathrm{a}}$ \\
\hline $\begin{array}{l}\text { EAC + Doxorubicin + } \\
\text { S. officinalis L. }\end{array}$ & $10.19 \pm 1.99^{\mathrm{a}}$ & $3.66 \pm 0.37^{\mathrm{a}}$ & $0.19 \pm 0.09^{a}$ & $2.21 \pm 0.43^{\mathrm{a}}$ \\
\hline
\end{tabular}

Normal values WBC 6-15 109\%/l (Uray, 1992)

$\left(=\mathrm{p}<0.05\right.$ as compared to control group; ${ }^{\mathrm{a}}=\mathrm{p}<0.05$ as compared to $\mathrm{EAC}$ group)

in adjuvant cancer therapy. To this end, studies should be continued.

Acknowledgments: This experimental study was supported by the research grant PN-III-P2 2.1-CI-2017-0242 / 2017.

Declaration of interests: The authors report no conflict of interest.

\section{References}

1. Fiore G., Nencini C., Cavallo F., Capasso A., Bader A., Giorgi G. (2006). In vitro antiproliferative effect of six Salvia species on human tumor cell lines. Phytotherapy Research, 20(8), 701-703.

2. Folkman J. (2006). Angiogenesis. Annu. Rev. Med., 57, 1-18.

3. Kamatou G.P.P., Makunga N.P., Ramogola W.P.N., Viljoen A.M. (2008). South African Salvia species: A review of biological activities and phytochemistry. Journal of Ethnopharmacology, 119, 664-672.

4. Keshavarz M., Bidmeshkipour A., Mostafaie A., Mansouri K., Mohammadi-Motlagh H.R. (2011). Antitumor activity of Salvia officinalis is due to its anti-angiogenic, antimigratory and anti-proliferative effect. Cell Journal, 12(4), 477-482.

5. Jedinak A., Muckova M., Kostalova D., Maliar T., Masterova I. (2006). Antiprotease and anti-metastatic activity of ursolic acid isolated from Salvia officinalis. Z. Naturforsch C., 61(11-12), 777-782.

6. Marian E., Vicaș L.G., Jurca T., Mureșan M., Pallag A., Stan R.L., Sevastre B., Diaconeasa Z., Ionescu C.M.L. (2018). Salvia offcinalis L. and Verbascum phlomoides L.: chemical, antimicrobial, antioxidant and antitumor investigations. Rev. Chim., 69(2), 365-370.

7. Murray J.C. (2001). Methods in molecular medicine: angiogenesis protocols. New Jersey: Humana press, 3-20.

8. Pandya N.M., Dhalla N.S., Santani D.D. (2006). Angiogenesis - a new target for future therapy. Vascul. Pharmacol., 44(5), 265-274.
9. Plank M.J., Sleeman B.D. (2003). Tumor - induced angiogenesis: a review. J. Theor. Med., 5(3-4), 137-153.

10. Privitera G., Luca T., Castorina S., Passanisi R., Ruberto G., Napoli E. (2019). Anticancer activity of Salvia officinalis essential oil and its principal constituents against hormone-dependent tumour cells. Asian Pac. J. Trop. Biomed., 9(1), 24-28.

11. Sarpataki O., Stan R.L., Hangan A.C., Olah N.K., SevastreBerghian A.C., Benedec D., Hanganu D., Sevastre B., Marcus I. (2016) Anticancer activity of Euonymus europaeus fruit extract on transplantable mouse tumor model. Bulletin UASVM, Veterinary Medicine, 73(1), 161-168.

12. Sevastre B., Sarpataki O., Stan R.L., Taulescu M., SevastreBerghian A.C., Olah N.K., Furtuna F., Hanganu D., Hangan A.C., Cenariu M., Bâldea I. (2017). Activity of Euonymus europaeus fruits extract on human melanoma cells. Farmacia, 65(1), 56-62.

13. Sevastre B., Sarpataki O., Olah N.K., Stan R.L., Taulescu M., Marcus I., Cătoi C. (2014). Antitumor effect of Euonymus Europaeus on Ehrlich tumor cells in vivo. Farmacia, 62(5), 907-917.

14. Stan R.L., Hangan A.C., Dican L., Sevastre B., Hanganu D., Cătoi C., Sarpataki O., Ionescu C.M. (2013). Comparative study concerning mistletoe viscotoxins antitumor activity. Acta Biologica Hungarica, 64(3), 279-288.

15. Tamrat T., Yesudass D.R. (2018). A review on anticancer activity of some plant derived compounds and their mode of action. Nat. Prod. Chem. Res., 6(4), 330.

16. Thorn C.F., Oshiro C., Marsh S., Hernandez-Boussard T., McLeod H., Klein T.E., Altman R.B. (2011). Doxorubicin pathways: pharmacodynamics and adverse effects. Pharmacogenet. Genomics, 21(7), 440-446.

17. Uray Z. (1992), Handbook of biological and physiological data in laboratory animals. Biology of the laboratory animal and comparative oncology. Oncology Institute, Cluj- Napoca, 19 (in Romanian).

18. *** Farmacopeea Română (2008), Ed. X, Ed. Medicală, București. 